\title{
Systemic immune responses are associated with molecular characteristics of circulating tumor cells in head and neck squamous cell carcinoma
}

\author{
HIROE TADA* , YURINO NAGATA* , HIDEYUKI TAKAHASHI, TOSHIYUKI MATSUYAMA, \\ SHOTA IDA, IKKO MITO and KAZUAKI CHIKAMATSU \\ Department of Otolaryngology-Head and Neck Surgery, Gunma University \\ Graduate School of Medicine, Maebashi, Gunma 3718511, Japan
}

Received January 22, 2021; Accepted April 21, 2021

DOI: $10.3892 / \mathrm{mco} .2021 .2309$

\begin{abstract}
Systemic immunity mediated by circulating immune cells may affect clinical features, as well as the characteristics of circulating tumor cells (CTCs) in patients with head and neck squamous cell carcinoma (HNSCC). The present study aimed to analyze the influence of circulating immune cells, using their markers, on clinical features to investigate the association between systemic immunity and the molecular characteristics of CTCs. Circulating immune-cell markers were associated with disease progression and clinical outcomes in patients with HNSCC. Meanwhile, there was no significant association between the presence of CTCs and systemic immune-related markers. Moreover, phosphatidylinositol-4,5-bisphosphate 3-kinase catalytic subunit a expression in CTCs was significantly associated with higher lymphocyte counts $(\mathrm{P}=0.035)$ and an increased prognostic nutrition index $(\mathrm{P}=0.0157)$. Patients with $\mathrm{CTCs}$ expressing $C D 47$ exhibited significantly higher neutrophil $(\mathrm{P}=0.0031)$ and monocyte $(\mathrm{P}=0.0016)$ counts. Patients with CTCs expressing programmed cell death 1 ligand 2 exhibited lower $\mathrm{C}$-reactive protein (CRP) levels $(\mathrm{P}=0.0271)$ and a decreased $\mathrm{CRP} /$ albumin ratio $(\mathrm{P}=0.0207)$. The current results suggested that the interaction between CTCs and circulating immune cells may provide survival advantages via molecular alterations to CTCs.
\end{abstract}

Correspondence to: Professor Kazuaki Chikamatsu, Department of Otolaryngology-Head and Neck Surgery, Gunma University Graduate School of Medicine, 3-39-22 Showa-machi, Maebashi, Gunma 3718511, Japan

E-mail: tikamatu@gunma-u.ac.jp

*Contributed equally

Key words: circulating tumor cell, head and neck squamous cell carcinoma, circulating immune cells, systemic immunity, molecular characteristics

\section{Introduction}

Recently developed immune checkpoint-blockade therapies have been demonstrated to have remarkable benefits for the treatment of patients with recurrent/metastatic head and neck squamous cell carcinoma (R/M HNSCC) $(1,2)$. Many studies have focused on achieving a deeper understanding of the interaction between tumor cells and associated immune cells within the tumor microenvironment, to not only predict but also improve immunotherapeutic responsiveness $(3,4)$. Similarly, in the bloodstream complex interactions between tumor cells and immune cells play an important role in the formation of distant metastases and in clinical outcomes (5-7).

Most tumor cells that enter the bloodstream through intravasation from primary sites die by anoikis induced by detachment from the extracellular matrix, the shearing force of blood pressure, or immune surveillance. However, some are able to survive in the bloodstream through phenotypic and functional alterations that confer resistance to environmental stress and are called circulating tumor cells (CTCs). Among the three forms of cell death mentioned, immune surveillance is the most complex and fluctuates depending on the patient's disease progression, nutritional status, and treatment pressure. In contrast, protumoral skewing of the immune system supports evasion of immune surveillance and promotes tumor cell dissemination. To date, the role of systemic immunity in cancer patients has been exclusively investigated and reported with regard to its clinical significance $(8,9)$; however, the relationship between systemic immunity and CTCs remains unclear. Interestingly, in patients with breast cancer, the presence of CTCs has been correlated with a reduction in $\mathrm{CD}^{+}, \mathrm{CD}^{+}$, and $\mathrm{CD}^{+} \mathrm{T}$ cells (10). Another study in lung-cancer patients showed that numbers of CTCs were negatively correlated with those of $\mathrm{CD}^{+}, \mathrm{CD}^{+}$, and $\mathrm{CD}^{+} / \mathrm{CD}^{+} \mathrm{T}$ cells (11). However, further studies are needed to more precisely evaluate the impact of systemic immunity on CTCs in the bloodstream.

To address this issue, we first investigated whether the proportions of circulating immune cells were correlated with clinical features. We then analyzed the potential correlations with the molecular characteristics of CTCs. 


\section{Materials and methods}

Patients. This study enrolled 44 patients with untreated HNSCC from a previous study (12). Their median age was 66 years (range: 47-86). The tumor origins included the oral cavity $(n=4)$, nasopharynx $(n=2)$, oropharynx $(n=17)$, hypopharynx $(n=14)$, larynx $(n=4)$, and nasal cavity $(n=3)$. We evaluated several clinical variables, including age, $T$ factor, $\mathrm{N}$ factor, stage, locoregional recurrence, distant metastasis, initial treatment response, and presence of CTCs. This study was approved by the Ethical Committee of Gunma University Hospital (no. 12-12), and written informed consent was obtained from each patient.

Data acquisition and systemic immune-related markers. Laboratory data, including neutrophil, lymphocyte, monocyte, and platelet counts, as well as serum C-reactive protein (CRP) and albumin levels, were collected from patients' clinical records within 2 weeks of blood collection for CTC isolation. The neutrophil-lymphocyte ratio (NLR), platelet-lymphocyte ratio (PLR), and lymphocyte-monocyte ratio (LMR) were calculated by dividing the absolute values of the corresponding hematological parameters. The systemic immune-inflammation index (SII), prognostic nutrition index (PNI), and CRP/albumin ratio (CAR) were calculated as: $\mathrm{SII}=($ platelet count $\mathrm{x}$ neutrophil count $) /$ lymphocyte count $(13), \mathrm{PNI}=(10 \mathrm{x}$ serum albumin $)+(0.005 \mathrm{x}$ lymphocyte count) (14), and CAR = CRP/albumin (15).

CTC detection and gene expression. CTC detection and molecular data from a previous study were used (12). Blood samples were collected from 44 patients with untreated HNSCC; CTCs were isolated using a CellSieve ${ }^{\mathrm{TM}}$ microfilter (Creatv MicroTech, Inc.). In brief, peripheral blood samples $(7.5 \mathrm{ml})$ were passed through a CellSieve ${ }^{\mathrm{TM}}$ microfilter. The filter was then washed with phosphate-buffered saline three times and transferred into a new tube, labeled as CTCs. The first filtrate was passed through a second filter to capture control leukocytes. The second filter was washed, transferred into another tube, and used as a control.

Total RNA from the CTCs was extracted using an RNeasy Micro Kit (Qiagen, Inc.) according to the manufacturer's instructions. Complementary DNA synthesis was performed using the QuantiTect Reverse Transcription kit (Qiagen, Inc.) with 14 cycles of preamplification using the TaqMan $^{\mathrm{TM}}$ PreAmp Master Mix Kit (Applied Biosystems). The products were analyzed using the real-time quantitative polymerase chain reaction (Applied Biosystems). Primers for the 14 target genes epithelial cell adhesion molecule [EPCAM: Hs00158980_m1], MET: Hs01565576_m1, keratin 19 [KRT19: Hs00761767_s1], epidermal growth factor receptor [EGFR: Hs01076090_m1], phosphatidylinositol-4,5-bisphosphate 3-kinase catalytic subunit alpha [PIK3CA: Hs00907957_m1], cyclin D1 [CCND1: Hs00765553_ $\mathrm{m} 1]$, snail family transcriptional repressor 1 [SNAII: Hs00195591_m1], vimentin [VIM: Hs00958111_m1], CD44: Hs01075861_m1, nanog homeobox [NANOG: Hs04399610_g1], aldehyde dehydrogenase 1 family member A1 [ALDH1A1: Hs00946916_m1], CD47: Hs00179953_m1, CD274: Hs01125301_m1, and programmed cell death 1 ligand 2
[PDCD1LG2: Hs01057777_m1] and ACTB (Hs01060665_g1, normalization control) were purchased from Applied Biosystems (TaqMan ${ }^{\mathrm{TM}}$ Gene Expression Assays). CTC gene expression levels were determined using a relative quantification method. Detection of at least one of the four epithelial-related genes (EPCAM, MET, KRT19, and EGFR) was defined as CTC positivity. The cycle threshold $(\mathrm{Ct})$ values of the target genes were normalized to those of the reference gene ACTB. Expression levels were estimated as fold changes and compared to those in the control leukocyte group using the $2^{-\Delta \Delta C t}$ relative quantification method (16). When the $2^{-\Delta \Delta C t}$ value was $>1$, the sample was assessed as positive for the expression of the gene.

Statistical analysis. GraphPad Prism version 8.0 for Windows (GraphPad Software, Inc.) was used for all analyses. Mann-Whitney $\mathrm{U}$ tests were used to assess differences in continuous variables. Kaplan-Meier curves were plotted and compared using log-rank tests to compare survival curves between subgroups. The optimal cutoff values of circulating immune cells as well as their related markers for progression-free survival (PFS) and overall survival (OS) were determined based on receiver operating characteristic curve analysis. Two-sided P-values $<0.05$ were considered to be statistically significant.

\section{Results}

Clinical significance of systemic immune-related markers. A total of 44 treatment-naïve patients with HNSCC were enrolled in this study. Their characteristics are listed in Table I. Twenty-eight (63.6\%) of the 44 were positive for CTCs as described previously (12). First, we determined whether systemic immune-related markers were associated with clinical factors and the presence of CTCs. Monocyte counts in elderly patients were significantly higher than those in younger patients $(\mathrm{P}=0.0339)$. Patients with advanced-stage disease exhibited significantly higher monocyte counts and LMR values than those with early-stage disease. Interestingly, patients with distant metastases during the follow-up period had significantly lower lymphocyte counts, NLR, and LMR. Meanwhile, there was no significant correlation between the presence of CTCs and any systemic immune-related markers. Next, we analyzed the prognostic significance of systemic immune-related markers in these 44 patients (Table II). As expected, various systemic immune-related markers, including lymphocyte counts, NLR, PLR, LMR, SII, and PNI, were associated with PFS (lymphocyte counts, $\mathrm{P}=0.0021$; NLR, $\mathrm{P}=0.005$; PLR, $\mathrm{P}=0.04$; LMR, $\mathrm{P}=0.013$; SII, $\mathrm{P}=0.046$; and PNI, $\mathrm{P}=0.015)$. Monocyte counts and PLR were significantly associated with OS (monocyte counts, $\mathrm{P}=0.049$; PLR, $\mathrm{P}=0.0441)$. The corresponding Kaplan-Meier survival curves are shown in Fig. 1.

Association between systemic immune-related markers and the molecular characteristics of CTCs. Finally, we analyzed whether systemic immune-related markers were associated with specific molecular characteristics of CTCs. Gene expression tests revealed that the expression levels of five genes, PIK3CA, CD44, NANOG, CD47, and PDCDILG2, 


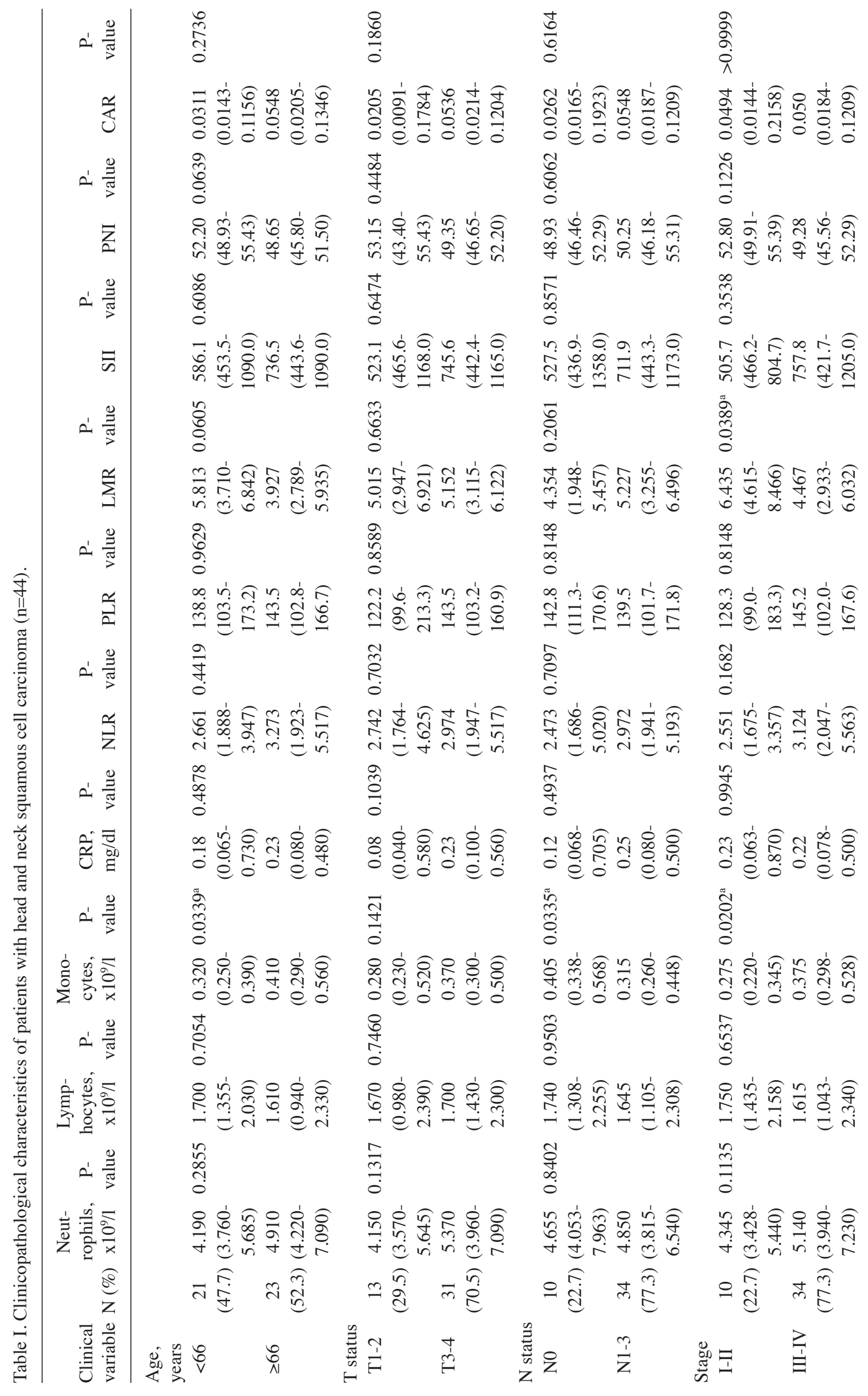




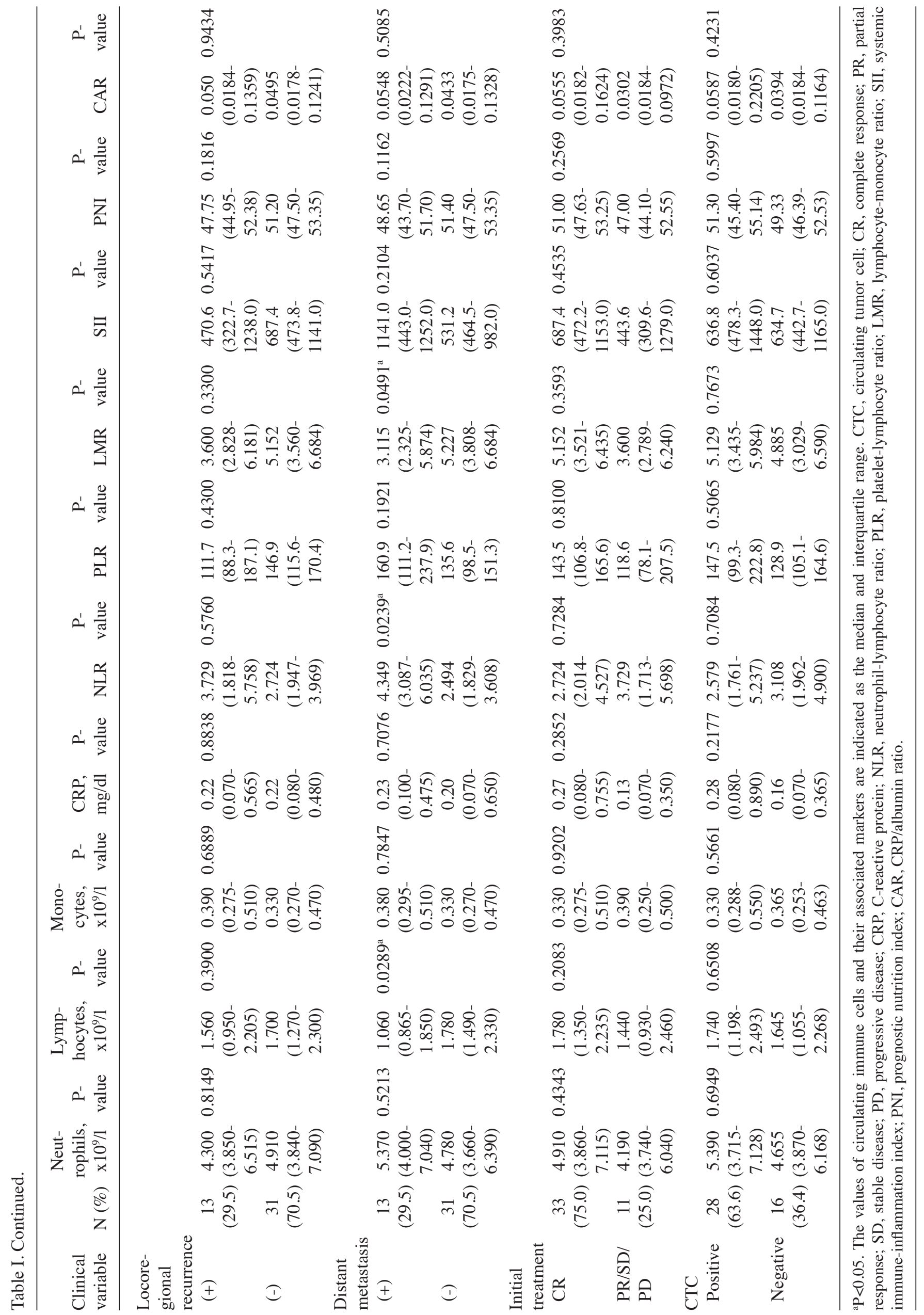


Table II. Prognostic value of systemic immune-related markers in patients with head and neck squamous cell carcinoma $(n=44)$.

\begin{tabular}{|c|c|c|c|c|}
\hline Variables & $\mathrm{N}$ & P-value & HR & $95 \% \mathrm{CI}$ \\
\hline \multicolumn{5}{|c|}{ Neutrophils, x $10^{9} / 1$} \\
\hline$<3.670$ & 8 & 0.1919 & 3.5330 & $1.0010-12.4800$ \\
\hline$\geq 3.670$ & 36 & & & \\
\hline \multicolumn{5}{|c|}{ Lymphocytes, x109/1 } \\
\hline$<1.090$ & 10 & $0.0021^{\mathrm{a}}$ & 0.2339 & $0.0608-0.9001$ \\
\hline$\geq 1.090$ & 34 & & & \\
\hline \multicolumn{5}{|c|}{ Monocytes, x109/1 } \\
\hline$<0.375$ & 25 & 0.2740 & 1.7460 & $0.6204-4.9160$ \\
\hline$\geq 0.375$ & 19 & & & \\
\hline \multicolumn{5}{|l|}{ CRP, mg/dl } \\
\hline$<0.065$ & 36 & 0.1440 & 0.2489 & $0.0738-0.8397$ \\
\hline$\geq 0.065$ & 8 & & & \\
\hline \multicolumn{5}{|l|}{ NLR } \\
\hline$<3.710$ & 28 & $0.0050^{\mathrm{a}}$ & 4.1040 & $1.3960-12.0600$ \\
\hline$\geq 3.710$ & 16 & & & \\
\hline \multicolumn{5}{|l|}{ PLR } \\
\hline$<157.1$ & 30 & $0.0400^{\mathrm{a}}$ & 2.7610 & $0.9080-8.3980$ \\
\hline$\geq 157.1$ & 14 & & & \\
\hline \multicolumn{5}{|l|}{ LMR } \\
\hline$<3.704$ & 15 & $0.0130^{\mathrm{a}}$ & 0.2951 & $0.0985-0.8842$ \\
\hline$\geq 3.704$ & 29 & & & \\
\hline \multicolumn{5}{|l|}{ SII } \\
\hline$<1,027$ & 30 & $0.0460^{\mathrm{a}}$ & 2.6870 & $0.8890-8.1210$ \\
\hline$\geq 1,027$ & 14 & & & \\
\hline \multicolumn{5}{|l|}{ PNI } \\
\hline$<49.43$ & 21 & $0.0150^{\mathrm{a}}$ & 0.2697 & $0.0969-0.7504$ \\
\hline$\geq 49.43$ & 23 & & & \\
\hline \multicolumn{5}{|l|}{ CAR } \\
\hline$<0.01745$ & 9 & 0.1010 & 4.6300 & $1.4350-14.9300$ \\
\hline$\geq 0.01745$ & 35 & & & \\
\hline
\end{tabular}

B, Overall survival

\begin{tabular}{|c|c|c|c|c|}
\hline Variables & $\mathrm{N}$ & P-value & HR & $95 \% \mathrm{CI}$ \\
\hline
\end{tabular}

Neutrophils, $\mathrm{x} 10^{9} / 1$

$\begin{array}{llll}<4.000 & 13 & 0.1053 & \text { Undefined Undefined } \\ \geq 4.000 & 31 & & \end{array}$

Lymphocytes, $\mathrm{x} 10^{9} / 1$

$\begin{array}{lllll}<1.465 & 15 & 0.2465 & 0.4250 & 0.0910-1.9860 \\ \geq 1.465 & 29 & & & \end{array}$

Monocytes, $\mathrm{x} 10^{9} / 1$

$\begin{array}{lllll}<0.375 & 25 & 0.0490^{\mathrm{a}} & 4.4270 & 0.9467-20.7000 \\ \geq 0.375 & 19 & & & \end{array}$

CRP, mg/dl

$<0.065$

$\geq 0.065$

$36 \quad 0.1320$ Undefined Undefined
Table II. Continued.

B, Overall survival

\begin{tabular}{lrrrr}
\hline Variables & N & P-value & HR & $95 \%$ CI \\
\hline NLR & & & & \\
$<3.710$ & 28 & 0.1090 & 3.4560 & $0.7702-15.5000$ \\
$\geq 3.710$ & 16 & & & \\
PLR & & & & \\
$<157.1$ & 30 & $0.0441^{\mathrm{a}}$ & 4.4910 & $0.9573-21.0700$ \\
$\geq 157.1$ & 14 & & & \\
LMR & & & & \\
$<6.496$ & 35 & 0.2147 & Undefined & Undefined \\
$\geq 6.496$ & 9 & & & \\
SII & & & & \\
$<836$ & 27 & 0.0975 & 3.5890 & $0.7962-16.1800$ \\
$\geq 836$ & 17 & & & \\
PNI & & & & \\
$<43.63$ & 5 & 0.6657 & 0.7170 & $0.1209-4.2520$ \\
$\geq 43.63$ & 39 & & & \\
CAR & & & & \\
$<0.01745$ & 95 & 0.1060 & Undefined & Undefined \\
$\geq 0.01745$ & 35 & & & \\
\hline
\end{tabular}

${ }^{\text {a }}<<0.05$. HR, hazard ratio; CI, confidence interval; CRP, C-reactive protein; NLR, neutrophil-lymphocyte ratio; PLR, platelet-lymphocyte ratio; LMR, lymphocyte-monocyte ratio; SII, systemic immune-inflammation index; PNI, prognostic nutrition index; CAR, CRP/albumin ratio.

were significantly correlated with systemic immune-related markers (Table III). In particular, PIK3CA expression in CTCs was significantly correlated with higher lymphocyte counts $(\mathrm{P}=0.035)$ and $\mathrm{PNI}(\mathrm{P}=0.0157)$. Patients with $\mathrm{CTC}$ s expressing CD47 showed significantly higher neutrophil $(\mathrm{P}=0.0031)$ and monocyte counts $(\mathrm{P}=0.0016)$. Furthermore, those with CTCs expressing PDCDILG2 showed significantly lower CRP $(\mathrm{P}=0.0271)$ and CAR $(\mathrm{P}=0.0207)$ levels.

\section{Discussion}

Circulating immune-related cells extravasate, migrate toward the tumor site, and perform multiple important functions in the immune response against tumor cells. It is well known that the interaction between immune cells and tumor cells in the tumor microenvironment is associated with prognosis and treatment efficacy $(3,4)$. Systemic immunity in cancer patients also plays a crucial role in processes ranging from tumor initiation to metastatic progression. To date, several systemic immune-related markers, including NLR, PLR, and SII, have been reported to reflect disease progression and therapeutic response as well as predict prognosis in patients with HNSCC (17-19). In the present study, despite its small sample size, several immune-related markers were clearly correlated with clinical features and 
A

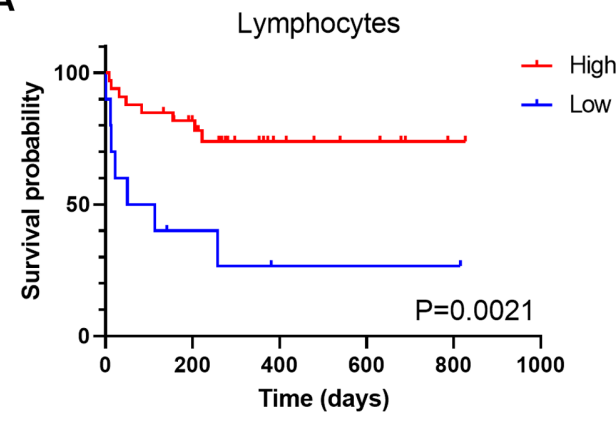

PLR

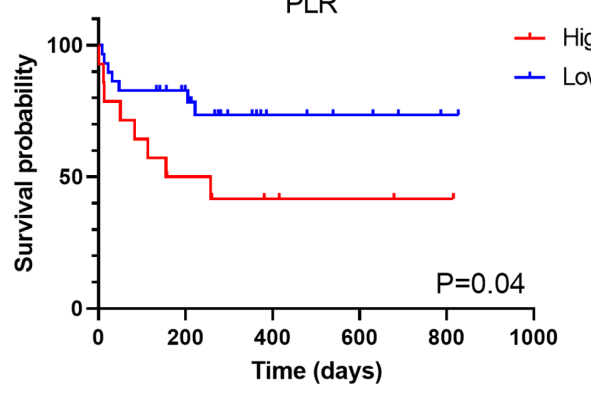

SII

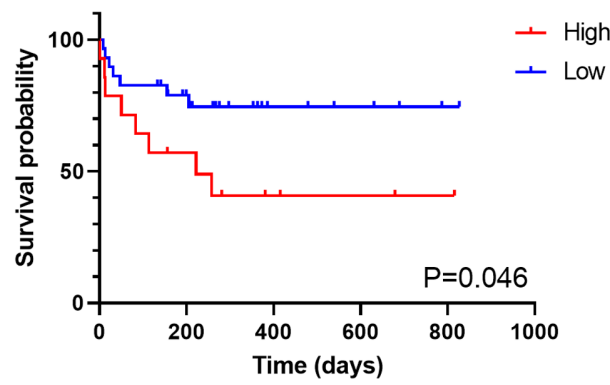

B

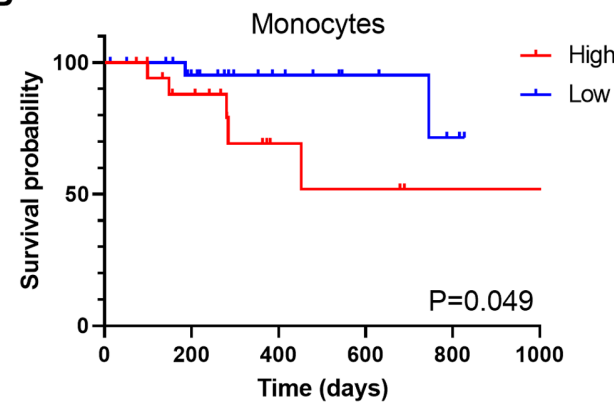

NLR

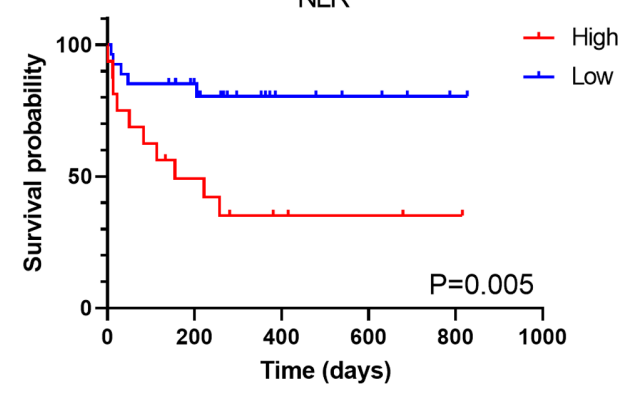

LMR

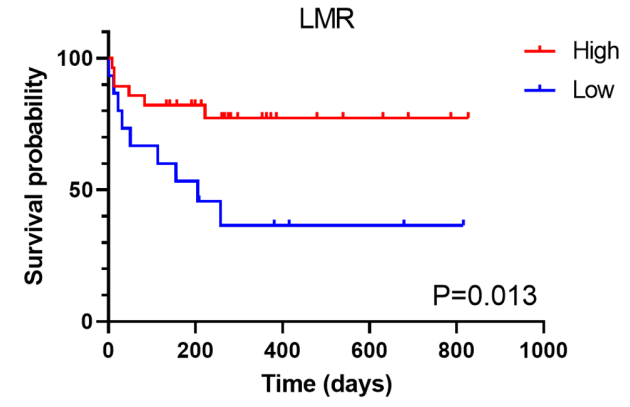

PNI
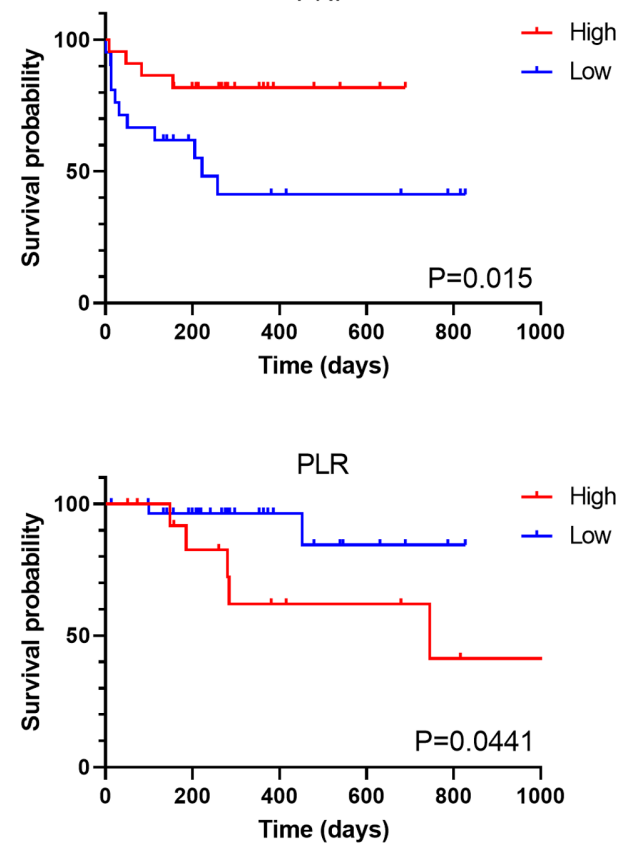

Figure 1. Kaplan-Meier survival analysis in patients with head and neck squamous cell carcinoma according to circulating immune cells and their associated markers. (A) Progression-free survival. (B) Overall survival. Optimal cut-off values were determined by receiver operating characteristic curve analysis. NLR, neutrophil-lymphocyte ratio; PLR, platelet-lymphocyte ratio; LMR, lymphocyte-monocyte ratio; SII, systemic immune-inflammation index; PNI, prognostic nutrition index.

prognosis. Patients with advanced disease exhibited significantly higher monocyte counts and lower LMR than those with early-stage disease. Our previous study on peripheral monocytes in patients with oropharyngeal squamous cell carcinoma (OPSCC) indicated that elevated monocyte counts and lower LMR were independent prognostic factors for PFS and OS, respectively (20). In line with our previous study, in cases of HNSCC including OPSCC, similar findings were observed, suggesting that circulating monocytes in patients with HNSCC are closely related to disease status. Although the reason for this association is not yet fully understood, a high monocy te count generally reflects chronic inflammatory conditions, which can promote angiogenesis, induce cell proliferation, increase reactive oxygen species production, and suppress antitumor immunity $(21,22)$. In our previous study, the presence of CTCs was associated with treatment response, locoregional recurrence, and PFS (12); however, there was no significant correlation between the presence of CTCs and systemic immune-related markers. Therefore, systemic immune-related markers may be insufficient to assess antitumor immunity against CTCs. Notably, lymphocyte-related markers, lymphocyte counts, NLR, and 


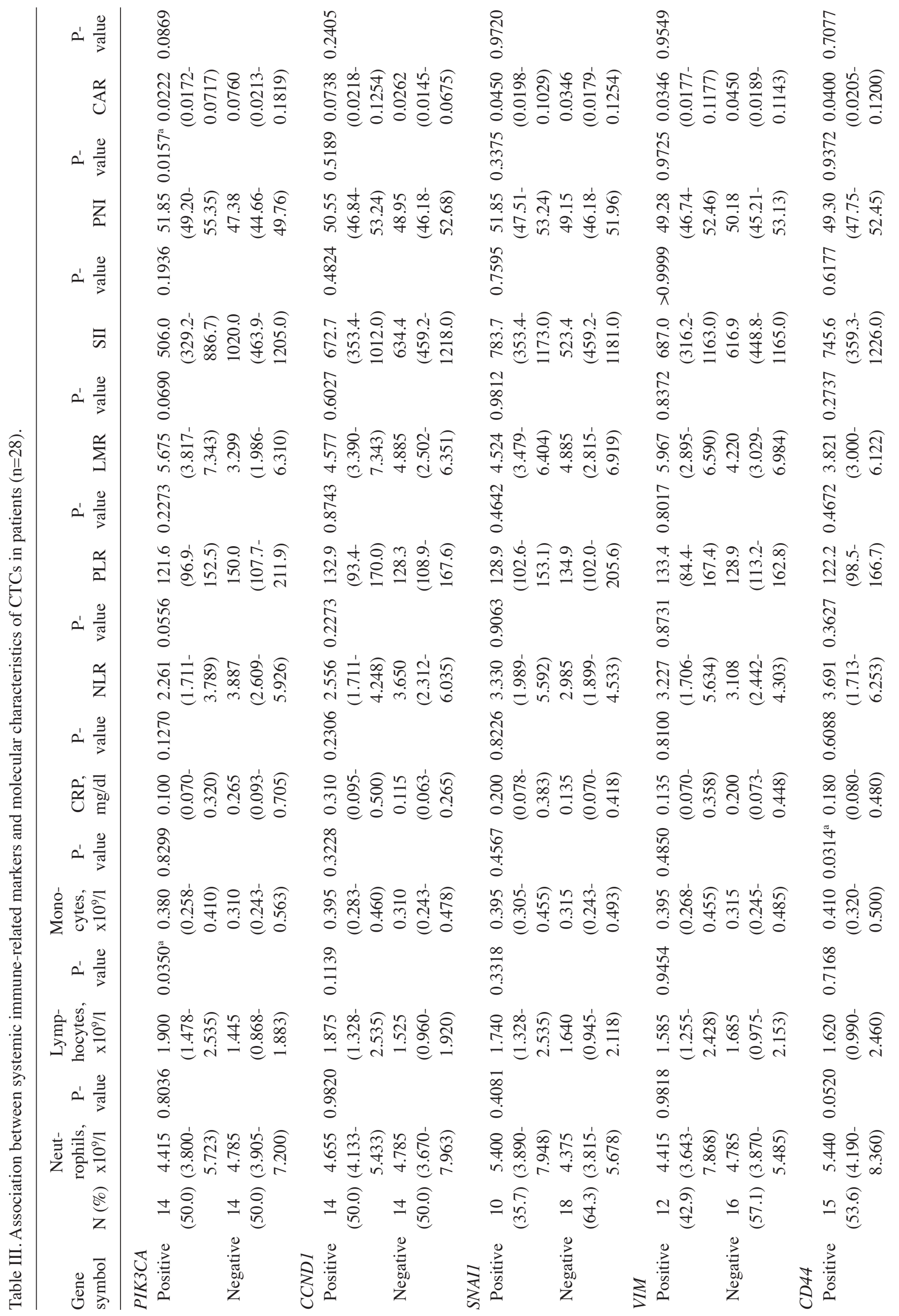




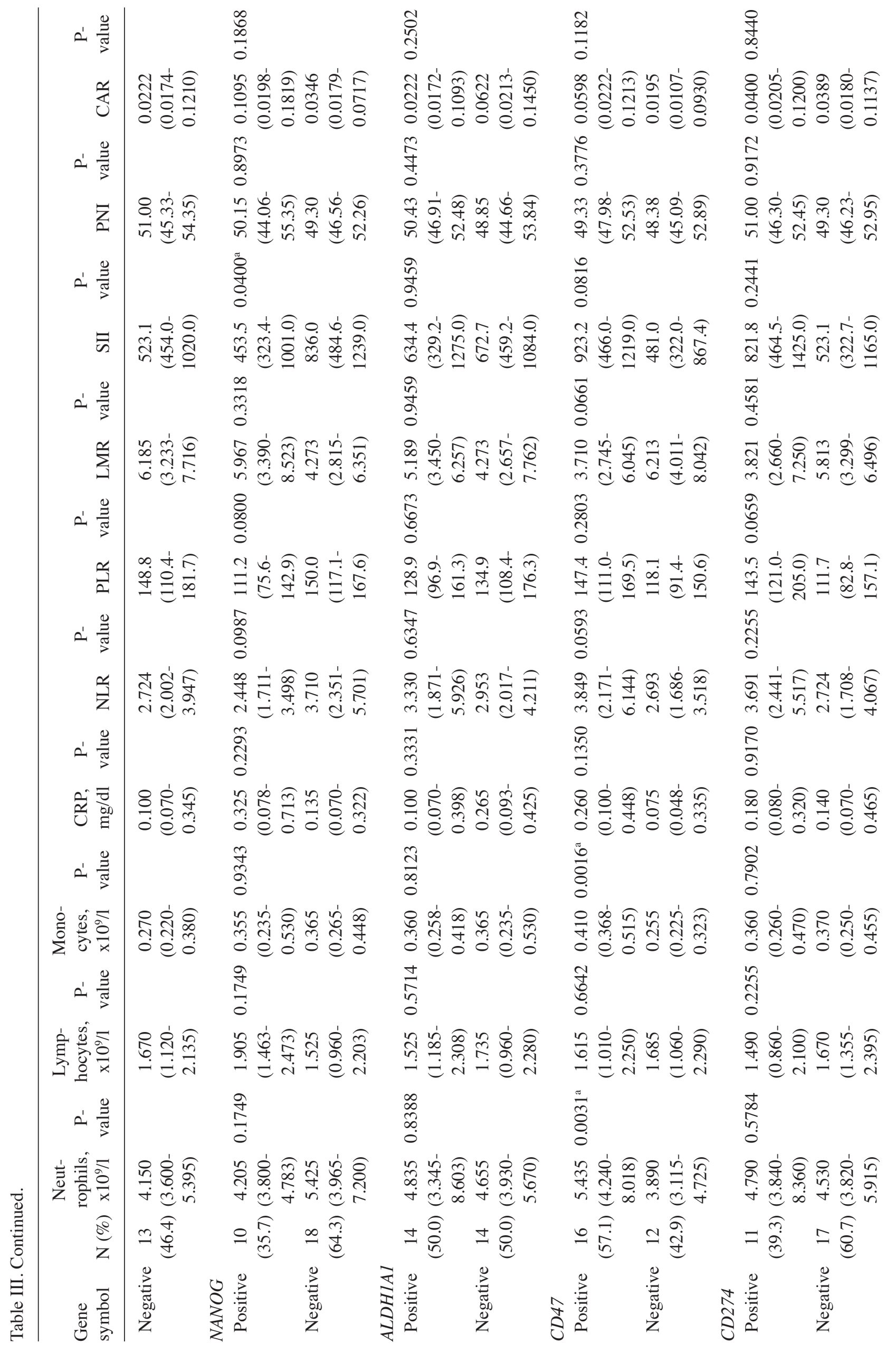




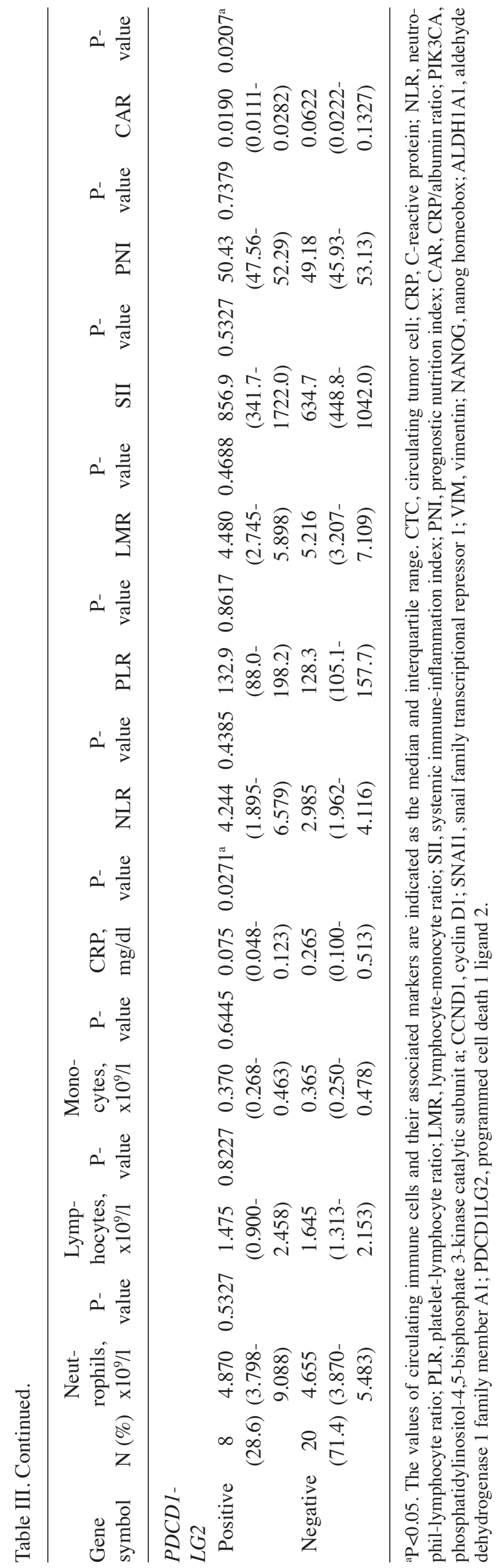

LMR were significantly associated with distant metastasis. These findings imply that lymphocyte-mediated systemic immune responses may contribute to the prevention of distant metastasis. So far, several studies have investigated the relationship between systemic immune-related markers and primary tumor characteristics using immunohistochemistry or molecular analysis; however, it seems that no meaningful correlations have yet been observed (23-25). In the dynamic multistep process of colonization of distant organs, CTCs that disseminate in the bloodstream and seed new tumors at distant organ sites might have acquired a status of dormancy in the peripheral bloodstream. Therefore, we investigated the relationship between the molecular characteristics of CTCs and systemic immune-related markers.

Among the 10 genes tested, PIK3CA,CD47, and $P D C D 1 L G 2$ expression levels in CTCs were significantly correlated with two systemic immune-related markers. PIK3CA is an oncogene that is known to play a role in regulating cell proliferation, invasion, and metabolism $(26,27)$. Chen et al demonstrated that PIK3CA overexpression promotes the epithelial-mesenchymal transition and enriches cancer stem cells in both murine and human HNSCC cell lines (28). Moreover, PIK3CA overexpression was reported to be associated with poor outcomes (29). Patients with PIK3CA-positive CTCs showed higher lymphocyte counts and higher PNI, suggesting that CTCs could acquire immune resistance through PIK3CA expression. Indeed, in mouse experiments using pancreatic cancer cell lines, PIK3CA-AKT signaling in tumors was found to reduce the cell-surface expression levels of major histocompatibility complex I molecules and CD80, which promote immune evasion (30). Additionally, a tendency toward significance between the other two lymphocyte-related markers (NLR and LMR) and PIK3CA expression in CTCs suggests immune evasion by PIK3CA expression in CTCs.

CD47-positive CTCs were found in patients with higher neutrophil and monocyte counts. In addition, high NLR, low LMR, and high SII showed a tendency toward $C D 47$ expression in CTCs. CD47 has been shown to be highly expressed in multiple cancer types, including HNSCC (31-34). We previously reported that $\mathrm{CD} 47$ is expressed in approximately half (56.8\%) of oral SCC tissues and that CD47 expression correlates with poor OS (34). Most importantly, CD47 acts as a signal to inhibit phagocytic activity by binding to signal regulatory protein $\alpha$ (SIRP $\alpha$ ) present on phagocytes (35); thus, it has been suggested that tumor cells evade the host's immune surveillance by expressing CD47 on their surface. As SIRP $\alpha$ is known to be expressed on monocytes, macrophages, neutrophils, and dendritic cells $(36,37)$, in cases of higher circulating monocyte and/or neutrophil counts, CTCs may be more likely to be phagocytosed and killed by these cells. Some CTCs may upregulate CD47 expression to evade the innate immune response mediated by neutrophils and monocytes.

Finally, PDCDILG2 expression in CTCs was observed in patients with lower CRP values and lower CARs. Similar to programmed cell death 1 ligand 1 (PD-L1), PD-L2 encoded by the PDCDIIG2 gene binds to PD-1 on T cells and inhibits T-cell proliferation and effector functions (38). Moreover, the expression of PD-L2 is regulated by interferon receptor 
signaling pathways, particularly the interferon- $\gamma($ IFN- $\gamma)$ pathway (39). CRP is an acute inflammatory protein that increases in response to infection and inflammation. Recently, Yoshida et al demonstrated that CRP inhibits proliferation, activation-associated phenotypes, and the effector function of activated T cells in patients with melanoma (40). Thus, high CRP levels may impair adaptive immunity and consequently affect PD-L2 expression on CTCs via reduced IFN- $\gamma$ production.

Although the relatively small sample size partially limits the strength of these findings, our results indicate that circulating immune cells and their related markers are correlated with disease progression and clinical outcomes in patients with HNSCC. Moreover, the interaction between CTCs and circulating immune cells appears to provide survival advantages via molecular alterations to CTCs. Further elucidation of the survival mechanisms of CTCs in the blood microenvironment may provide new insights into novel therapeutic strategies targeting CTCs.

\section{Acknowledgements}

Not applicable.

\section{Funding}

The present study was supported in part by a Grant-in-Aid for Scientific Research (grant nos. 20K18243, 20K09747, 19K18794, 19K18758 and 20H03834) from the Japan Ministry of Education, Culture, Sports, Science and Technology.

\section{Availability of data and materials}

The datasets used and/or analyzed during the current study are available from the corresponding author on reasonable request.

\section{Authors' contributions}

HTad and KC conceived and designed the study. HTak, YN, TM, SI and IM acquired the data. HTad and HTak analyzed and interpreted the data. HTad, HTak and KC confirm the authenticity of the raw data. HTad and $\mathrm{KC}$ wrote the manuscript. All authors have read and approved the final manuscript.

\section{Ethics approval and consent to participate}

The present study was approved by the Ethical Committee of Gunma University Hospital (Maebashi, Japan; approval no. 12-12), and written informed consent was obtained from each patient.

\section{Patient consent for publication}

Not applicable.

\section{Competing interests}

The authors declare that they have no competing interests.

\section{References}

1. Ferris RL, Blumenschein G Jr, Fayette J, Guigay J, Colevas AD, Licitra L, Harrington K, Kasper S, Vokes EE, Even C, et al: Nivolumab for recurrent squamous-cell carcinoma of the head and neck. N Engl J Med 375: 1856-1867, 2016.

2. Burtness B, Harrington KJ, Greil R, Soulières D, Tahara M, de Castro G Jr, Psyrri A, Basté N, Neupane P, Bratland A, et al: Pembrolizumab alone or with chemotherapy versus cetuximab with chemotherapy for recurrent or metastatic squamous cell carcinoma of the head and neck (KEYNOTE-048): A randomised, open-label, phase 3 study. Lancet 394: 1915-1928, 2019.

3. Pitt JM, Marabelle A, Eggermont A, Soria JC, Kroemer G and Zitvogel L: Targeting the tumor microenvironment: Removing obstruction to anticancer immune responses and immunotherapy. Ann Oncol 27: 1482-1492, 2016.

4. Murciano-Goroff YR, Warner AB and Wolchok JD: The future of cancer immunotherapy: Microenvironment-targeting combinations. Cell Res 30: 507-519, 2020.

5. Alečković M, McAllister SS and Polyak K: Metastasis as a systemic disease: Molecular insights and clinical implications. Biochim Biophys Acta Rev Cancer 1872: 89-102, 2019.

6. Diakos CI, Charles KA, McMillan DC and Clarke SJ: Cancer-related inflammation and treatment effectiveness. Lancet Oncol 15: e493-e503, 2014.

7. Janssen LME, Ramsay EE, Logsdon CD and Overwijk WW: The immune system in cancer metastasis: Friend or foe? J Immunother Cancer 5: 79, 2017.

8. Dolan RD, Laird BJA, Horgan PG and McMillan DC: The prognostic value of the systemic inflammatory response in randomised clinical trials in cancer: A systematic review. Crit Rev Oncol Hematol 132: 130-137, 2018.

9. Gutkin DW and Shurin MR: Clinical evaluation of systemic and local immune responses in cancer: Time for integration. Cancer Immunol Immunother 63: 45-57, 2014.

10. Mego M, Gao H, Cohen EN, Anfossi S, Giordano A, Sanda T, Fouad TM, De Giorgi U, Giuliano M, Woodward WA, et al: Circulating tumor cells (CTC) are associated with defects in adaptive immunity in patients with inflammatory breast cancer. J Cancer 7: 1095-1104, 2016.

11. Ye L, Zhang F, Li H, Yang L, Lv T, Gu W and Song Y: Circulating tumor cells were associated with the number of T lymphocyte subsets and NK cells in peripheral blood in advanced non-small-cell lung cancer. Dis Markers 2017: $5727815,2017$.

12. Tada H, Takahashi H, Kuwabara-Yokobori Y, Shino M and Chikamatsu K: Molecular profiling of circulating tumor cells predicts clinical outcome in head and neck squamous cell carcinoma. Oral Oncol 102: 104558, 2020.

13. Hu B, Yang XR, Xu Y, Sun YF, Sun C, Guo W, Zhang X, Wang WM, Qiu SJ, Zhou J and Fan J: Systemic immune-inflammation index predicts prognosis of patients after curative resection for hepatocellular carcinoma. Clin Cancer Res 20: 6212-6222, 2014.

14. Onodera T, Goseki N and Kosaki G: Prognostic nutritional index in gastrointestinal surgery of malnourished cancer patients. Nihon Geka Gakkai Zasshi 85: 1001-1005, 1984 (In Japanese).

15. Ranzani OT, Zampieri FG, Forte DN, Azevedo LC and Park M: C-reactive protein/albumin ratio predicts 90 -day mortality of septic patients. PLoS One 8: e59321, 2013.

16. Livak KJ and Schmittgen TD: Analysis of relative gene expression data using real-time quantitative PCR and the 2(-Delta Delta C(T)) method. Methods 25: 402-408, 2001.

17. Jiang W, Chen Y, Huang J, Xi D, Chen J, Shao Y, Xu G, Ying W, Wei J, Chen J, et al: Systemic immune-inflammation index predicts the clinical outcome in patients with nasopharyngeal carcinoma: A propensity score-matched analysis. Oncotarget 8: 66075-66086, 2017.

18. Diao P, Wu Y, Li J, Zhang W, Huang R, Zhou C, Wang Y and Cheng J: Preoperative systemic immune-inflammation index predicts prognosis of patients with oral squamous cell carcinoma after curative resection. J Transl Med 16: 365, 2018.

19. Szilasi Z, Jósa V, Zrubka Z, Mezei T, Vass T, Merkel K, Helfferich F and Baranyai Z: Neutrophil-to-lymphocyte and platelet-to-lymphocyte ratios as prognostic markers of survival in patients with head and neck tumours-results of a retrospective multicentric study. Int J Environ Res Public Health 17: 1742, 2020 . 
20. Takahashi H, Sakakura K, Tada H, Kaira K, Oyama T and Chikamatsu K: Prognostic significance and population dynamics of peripheral monocytes in patients with oropharyngeal squamous cell carcinoma. Head Neck 41: 1880-1888, 2019.

21. Grivennikov SI, Greten FR and Karin M: Immunity, inflammation, and cancer. Cell 140: 883-899, 2010.

22. Greten FR and Grivennikov SI: Inflammation and cancer: Triggers, mechanisms, and consequences. Immunity 51: 27-41, 2019.

23. Chen ZY, Raghav K, Lieu CH, Jiang ZQ, Eng C, Vauthey JN, Chang GJ, Qiao W, Morris J, Hong D, et al: Cytokine profile and prognostic significance of high neutrophil-lymphocyte ratio in colorectal cancer. Br J Cancer 112: 1088-1097, 2015.

24. Yersal Ö, Cetinkünar S, Aktimur R, Aziret M, Özdas S, Erdem H and Yildirim K: Neutrophil/lymphocyte and platelet/lymphocyte ratios are not different among breast cancer subtypes. Asian Pac J Cancer Prev 18: 2227-2231, 2017.

25. Cao Z, Ji J, Zhang C, Wang F, Xu H, Yu Y and Sun Y: The preoperative neutrophil-to-lymphocyte ratio is not a marker of prostate cancer characteristics but is an independent predictor of biochemical recurrence in patients receiving radical prostatectomy. Cancer Med 8: 1004-1012, 2019.

26. Samuels $Y$ and Ericson $K$ : Oncogenic PI3K and its role in cancer. Curr Opin Oncol 18: 77-82, 2006.

27. Rogers SJ, Box C, Harrington KJ, Nutting C, Rhys-Evans $P$ and Eccles SA: The phosphoinositide 3-kinase signalling pathway as a therapeutic target in squamous cell carcinoma of the head and neck. Expert Opin Ther Targets 9: 769-790, 2005.

28. Chen X, Cao Y, Sedhom W, Lu L, Liu Y, Wang H, Oka M, Bornstein S, Said S, Song J and Lu SL: Distinct roles of PIK3CA in the enrichment and maintenance of cancer stem cells in head and neck squamous cell carcinoma. Mol Oncol 14: 139-158, 2020

29. García-Escudero R, Segrelles C, Dueñas M, Pombo M Ballestín C, Alonso-Riaño M, Nenclares P, Álvarez-Rodríguez R, Sánchez-Aniceto G, Ruíz-Alonso A, et al: Overexpression of PIK3CA in head and neck squamous cell carcinoma is associated with poor outcome and activation of the YAP pathway. Oral Oncol 79: 55-63, 2018.

30. Sivaram N, McLaughlin PA, Han HV, Petrenko O, Jiang YP, Ballou LM, Pham K, Liu C, van der Velden AW and Lin RZ: Tumor-intrinsic PIK3CA represses tumor immunogenecity in a model of pancreatic cancer. J Clin Invest 129: 3264-3276, 2019.
31. Willingham SB, Volkmer JP, Gentles AJ, Sahoo D, Dalerba P, Mitra SS, Wang J, Contreras-Trujillo H, Martin R, Cohen JD, et al: The CD47-signal regulatory protein alpha (SIRPa) interaction is a therapeutic target for human solid tumors. Proc Natl Acad Sci USA 109: 6662-6667, 2012.

32. Baccelli I, Stenzinger A, Vogel V, Pfitzner BM, Klein C, Wallwiener M, Scharpff M, Saini M, Holland-Letz T, Sinn HP, et al: Co-expression of MET and CD47 is a novel prognosticator for survival of luminal breast cancer patients. Oncotarget 5: 8147-8160, 2014.

33. Wang H, Tan M, Zhang S, Li X, Gao J, Zhang D, Hao Y, Gao S, Liu J and Lin B: Expression and significance of CD44, CD47 and c-met in ovarian clear cell carcinoma. Int J Mol Sci 16: 3391-3404, 2015

34. Sakakura K, Takahashi H, Kaira K, Toyoda M, Murata T, Ohnishi H, Oyama T and Chikamatsu K: Relationship between tumor-associated macrophage subsets and CD47 expression in squamous cell carcinoma of the head and neck in the tumor microenvironment. Lab Invest 96: 994-1003, 2016.

35. Matozaki T, Murata Y, Okazawa H and Ohnishi H: Functions and molecular mechanisms of the CD47-SIRPalpha signalling pathway. Trends Cell Biol 19: 72-80, 2009.

36. Adams S, van der Laan LJ, Vernon-Wilson E, Renardel de Lavalette C, Döpp EA, Dijkstra CD, Simmons DL and van den Berg TK: Signal-regulatory protein is selectively expressed by myeloid and neuronal cells. J Immunol 161: 1853-1859, 1998.

37. Kharitonenkov A, Chen Z, Sures I, Wang H, Schilling J and Ullrich A: A family of proteins that inhibit signalling through tyrosine kinase receptors. Nature 386: 181-186, 1997.

38. Rozali EN, Hato SV, Robinson BW, Lake RA and Lesterhuis WJ: Programmed death ligand 2 in cancer-induced immune suppression. Clin Dev Immunol 2012: 656340, 2012.

39. Garcia-Diaz A, Shin DS, Moreno BH, Saco J, Escuin-Ordinas H, Rodriguez GA, Zaretsky JM, Sun L, Hugo W, Wang X, et al: Interferon receptor signaling pathways regulating PD-L1 and PD-L2 expression. Cell Rep 19: 1189-1201, 2017.

40. Yoshida T, Ichikawa J, Giuroiu I, Laino AS, Hao Y, Krogsgaard M, Vassallo M, Woods DM, Stephen Hodi F and Weber J: C reactive protein impairs adaptive immunity in immune cells of patients with melanoma. J Immunother Cancer 8: e000234, 2020. 